\title{
Pervasiveness of exoribonuclease-resistant RNAs in plant viruses suggests new roles for these conserved RNA structures
}

\author{
Anna-Lena Steckelberg ${ }^{\star}$, Quentin Vicens $^{\mathrm{\&} *}$, and Jeffrey S. Kieft ${ }^{*}$
}

Department of Biochemistry and Molecular Genetics, and RNA BioScience Initiative, University of Colorado Denver School of Medicine, Aurora, CO 80045, USA.

anna-lena.steckelberg@ucdenver.edu; quentin.vicens@ucdenver.edu; jeffrey.kieft@ucdenver.edu

\& these authors contributed equally to this work

*corresponding authors 


\section{ABSTRACT}

2 Exoribonuclease-resistant RNAs (xrRNAs) are discrete folded RNA elements that block the processive

3 degradation of RNA by exoribonucleases. xrRNAs found in the $3^{\prime}$ untranslated regions (UTRs) of animal-

4 infecting flaviviruses and in all three members of the plant-infecting Dianthovirus adopt a complex ring-

$5 \quad$ like fold that blocks the exoribonuclease; this ability gives rise to viral non-coding subgenomic RNAs.

6 The degree to which these folded RNA elements exist in other viruses and in diverse contexts has been

7 unclear. Using computational tools and biochemical assays, we discovered that xrRNA elements are

8 widely found in viruses belonging to the Tombusviridae and Luteoviridae families of plant-infecting RNA

9 viruses, demonstrating their importance and widespread utility. Unexpectedly, many xrRNAs are located

10 in intergenic regions rather than in the $3^{\prime}$ UTR and some are associated with the $5^{\prime}$ ends of subgenomic

11 RNAs with protein-coding potential, suggesting that xrRNAs with similar scaffolds are involved in the

12 maturation or maintenance of diverse subgenomic RNAs, not just the ones generated from the 3'UTR. 


\section{INTRODUCTION}

During infection, positive-sense RNA viruses produce full-length genomic RNA and many produce subgenomic RNAs (sgRNA) that can encode viral proteins or act as "riboregulators" that interact with and influence the cellular and viral machinery to benefit viral infection ${ }^{1-6}$. Most viral sgRNAs are thought to be produced directly through transcription; however, recent discoveries show that some noncoding viral sgRNAs result from discrete RNA elements that block the progression of $5^{\prime}$ to $3^{\prime}$ exoribonucleases (Figure 1) ${ }^{7-15}$. Discrete, compactly-folded exoribonuclease-resistant RNA (xrRNA) elements were first identified in mosquito-borne flaviviruses (e.g. Dengue virus, Zika virus, West Nile virus), where they protect the genome's $3^{\prime}$ untranslated region (UTR) from degradation ${ }^{8}$. The resultant decay intermediates accumulate and comprise biologically active viral non-coding sgRNAs (Figure 1) $8,9,12,16-21$.

Extensive functional and high-resolution structural studies show that mosquito-borne flaviviral xrRNA $\left(\mathrm{xrRNA}_{\mathrm{F}}\right)$ function is conferred by a specific three-dimensional fold containing an interwoven pseudoknot stabilized by extensive conserved secondary and tertiary interactions; this creates an unusual ring-like conformation that protectively wraps around the $5^{\prime}$ end of the RNA structure ${ }^{22,23}$. xrRNAs are found broadly within flaviviruses including those that are tick-borne, are specific to insects, or have no known vector ${ }^{15,24,25}$. Comparison of diverse $x_{R N A}$ sequences revealed two classes; class $\left.I_{(x r R N A}{ }_{F 1}\right)$ is exemplified by mosquito-borne flaviviruses while class II ( $\left.\mathrm{xrRNA}_{\mathrm{F} 2}\right)$ is found in diverse flaviviruses ${ }^{25}$. Although aligned xrRNAF2 sequences show patterns, their three-dimensional structures are unknown, as are the structures of recently reported xrRNAs from most other viral clades ${ }^{7,13}$.

Recently, we structurally and functionally characterized xrRNAs from the 3'UTRs of dianthoviruses, plant-infecting positive-sense RNA viruses in the Tombusviridae family; similar to the $\operatorname{xrRNA}_{F}$, they function to produce a non-coding RNA derived from the viral $3^{\prime}$ UTR ${ }^{10,26}$. Dianthoviral 
xrRNAs $\left(x R N A_{D}\right)$ also rely on a pseudoknot that forms a protective ring-like structure ${ }^{26}$, but they have

very different sequences and secondary structures compared to $\operatorname{xrRNA}_{\mathrm{F} 1}$ and the ring is formed by a

different set of interactions (Figure 1). Although $\mathrm{xrRNA}_{\mathrm{F}}$ elements pervade the flaviviruses with

associated sequence and structural diversity, $x_{R N A}$ have only been identified in the three closely related

members of the Dianthovirus genus. This raises the question of whether xrRNAs similar to xrRNA $A_{D}$ are

more widespread and diverse than currently known, and thus if they may be an underappreciated way to

produce or protect viral RNAs. Moreover, the only available xrRNAD crystal structure is in an "open"

conformation that likely represents a necessary folding intermediate before the pseudoknot forms ${ }^{26}$

(Figure 1). Thus, we still do not know the full repertoire of secondary and tertiary interactions required to

form and stabilize the exoribonuclease-resistant pseudoknot state of xrRNA $A_{D}$. The lack of diverse

$\mathrm{xrRNA}_{\mathrm{D}}$ sequences prevents conclusions about the role, prevalence, and structural diversity of this fold.

To begin to address these questions, we used a bioinformatic approach to identify more xrRNAD

sequences among plant viruses, identifying over 40 putative new xrRNAD-like elements in viruses

belonging to the Tombusviridae and Luteoviridae families. In vitro assays show that these elements are

indeed resistant to Xrn1 and analysis of these new xrRNAs reveals both conservation and variability.

Furthermore, the genomic location of these new xrRNAs suggests new roles in the generation of sgRNA

species that have protein-coding potential, providing evidence that xrRNA-based RNA maturation

pathways may be more widespread than previously anticipated.

\section{RESULTS AND DISCUSSION}

To search for xrRNAD-like elements, we used the Infernal software (Eddy lab), which enables screening of massive datasets of DNA sequences for conserved structure patterns with poor sequence conservation ${ }^{27}$. Because the Dianthovirus genus only comprises three members (Red clover necrotic mosaic virus (RCNMV), Sweet clover necrotic mosaic virus (SCNMV), and Carnation ringspot virus 
62 (CRSV) $)^{26}$, we expanded our search to other plant-infecting positive-sense RNA viruses. The initial 63 search within a library of viral reference genomes (see Methods) identified two potential sequences among Luteoviridae; Poleroviruses: wheat leaf yellowing-associated virus isolate JN-U3 (GenBank ID \# NC_035451; Infernal E-value $=0.00025$, score $=44.3)$ and sugarcane yellow leaf virus $($ GenBank \#NC_000874; Infernal E-value = 6.5, score = 24.2). With these sequences added to the alignment, subsequent searches identified $>40$ candidates within the public repository of all available sequences for

Tombusviridae and Luteoviridae, demonstrating how powerful this tool is for computationally identifying functional elements in viral RNAs ${ }^{28}$.

Alignment of the putative $x_{R N A}$-like elements evealed that their predicted secondary structures contain conserved helices P1, P2, and the pseudoknot, which are supported by covariation but have little sequence conservation (R-scape ${ }^{29}$ E-values for the 12 covarying base pairs in the stems and the pseudoknot are within $3 \cdot 10^{-4}-8.10^{-13}\left(95^{\text {th }}\right.$ percentile $\left.=1.10^{-12}\right)$; Figure $\left.2 \mathrm{~A}\right) . \mathrm{L} 1$ and L2B are $>97 \%$ conserved in sequence. In the case of L1 and L2B, this is consistent with their role in creating a specific folded motif that promotes pseudoknot formation ${ }^{26}$. Also, two of the three nucleotides immediately upstream of the $3^{\prime}$ side of the pseudoknot are $>97 \%$ conserved, but their role is not obvious from the crystal structure of the open state. Likewise, the non-Watson-Crick A8-G33 base pair identified in the crystal structure (Figure 1) cannot be reconciled with the predominant presence of $\mathrm{G}$ at position 8 and G/A at position 33 in all the other sequences. These observations support the previous assertion that the crystallized open state represents a folding intermediate and that structural adjustments and additional interactions are present in the "closed" pseudoknot state. genera of the Tombusviridae family, as well as members of the Polerovirus and Enamovirus genera of the 
sequences from viruses of both families using our established in vitro Xrn 1 resistance assay ${ }^{11}$.

Specifically, in vitro-transcribed and purified RNA sequences from opium poppy mosaic virus (OPMV),

Maize chlorotic mottle virus (MCMV), Potato leafroll virus (PLRV) Maize yellow dwarf virus-RMV

(MYDV-RMV) and Hubei polero-like virus 1 (HuPLV1) were challenged with recombinant Xrn1. All

RNAs stopped Xrn1 degradation similarly to RCNMV xrRNAD (Figure 3A, B), demonstrating that they

are authentic xrRNAs and do not require additional trans-acting proteins for function. Moreover,

mutations to disrupt the putative pseudoknot in the MCMV, PLRV and HuPLV1 xrRNAs abolished Xrn1

resistance, while compensatory mutations that restore pseudoknot base pairing rescued the activity

(Figure 3C-E). In addition, the mapped Xrn1 stop site is at the base of P1 in all newly identified xrRNAs,

matching the xrRNA stop site (Figure 3F-H, Supplementary Figure S2). Overall, the conserved

secondary structure (Figure 2B), the location of the exoribonuclease halt site, and the strict dependence on

the pseudoknot for Xrn1 resistance suggest that these newly-identified xrRNAs use a similar molecular

fold and mechanism as the $\mathrm{xrRNA}_{\mathrm{D}}$, thus we classify them as such, and hereafter refer to the class as

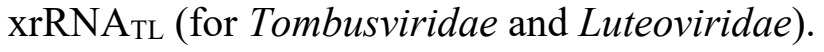

found in the Tombusviridae family (RCNMV, SCNMV, CRSV, OPMV, MCMV) possess a P3 stem-loop this part of the sequence is not required for Xrn 1 resistance by $\operatorname{xrRNA}_{\mathrm{TL}}$ in vitro ${ }^{26}$. Consistent with this, an analogous stem-loop $(\mathrm{P} 4)$ found in $\mathrm{xrRNA}_{\mathrm{F} 1}$ is also dispensable in vitro; the crystal structure indicates it may stabilize the pseudoknot through stacking interactions (Figure 1) ${ }^{22}$. Thus, in $\mathrm{xrRNA}_{\mathrm{TL}}$ coaxial stacking of $\mathrm{P} 3$ on $\mathrm{P} 1 / \mathrm{P} 2$ could help to stabilize the RNA structure in the cellular context during infection. 


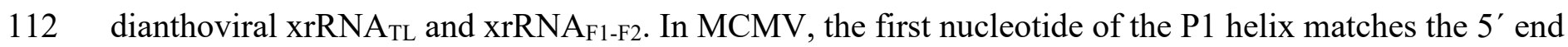

113 of sgRNA2 ${ }^{30}$, thus this new xrRNA element probably blocks Xrn1 to generate non-coding sgRNAs

114 derived from the 3'UTR, as with the dianthoviruses, flaviviruses, and other xrRNAs. However, for some

115 members of the Tombusviridae family as well as for Poleroviruses, xrRNATL is located in an intergenic

116 region, within 5-20 nt from the translation start site of ORF3a, and $135 \mathrm{nt}$ from the start site of a

117 readthrough protein encoded by ORF3-5 (our data suggest that ORF3a has not been annotated for all

118 Poleroviruses; Table S1). ORF3a codes for protein P3a, which is essential for long-distance movement of

119 the virus in plants ${ }^{31}$. Translation of ORF3a occurs from sgRNA1, generally at a non-AUG codon (Tables

1201 and S1) ${ }^{31-33}$. This implies that these xrRNAs, rather than functioning in non-coding RNA production,

121 act to produce or maintain protein coding RNAs (Figure 4); sgRNAs could be produced from full-length

122 genomic RNAs without requiring a subgenomic promoter, or sgRNAs produced by other means could be

123 protected from $5^{\prime}$ to $3^{\prime}$ degradation. Since the Tombusviridae and Luteoviridae families use $3^{\prime}$ proximal

124 cap-independent translation enhancers (3'-CITEs) to initiate translation, uncapped sgRNAs with xrRNAs

125 on their $5^{\prime}$ ends could still be translationally active ${ }^{34,35}$. Thus, these xrRNAs could be part of complex

126 translation regulation mechanisms involving these $3^{\prime}$-CITEs and different sgRNAs ${ }^{36}$.

127

128

129

130

131

132

133

134

135

136
Various roles for xrRNAs are possible, depending on their genetic context. The presence of xrRNAs in diverse locations within viral genomes suggests that new xrRNA scaffolds may emerge from analyzing sgRNA 5' termini from other viruses, as certainly not all xrRNA elements were identified by

the algorithm used here ${ }^{5,7,37}$ Intriguing candidates for novel xrRNA identification are viruses in which no obvious promoter elements for sgRNA production were identified, or viruses in which putative promoter sequences are downstream of the sgRNA $5^{\prime}$ end ${ }^{1,5,30,37}$. Many questions remain that pertain to understanding the structural/sequence requirements for $\mathrm{Xrn} 1$ resistance, the degree to which structural variation is tolerated, and how sequence diversity is integrated into similar folds ${ }^{38}$. The now-expanded set 
bioRxiv preprint doi: https://doi.org/10.1101/433672; this version posted October 2, 2018. The copyright holder for this preprint (which was not certified by peer review) is the author/funder. All rights reserved. No reuse allowed without permission.

137 of $x_{R N A}$ TL candidates provides a broader phylogeny for future bioinformatic and structural studies that 138 will address these points.

139 
correspond to positive hits but with a larger sequence or structure variation. By the time the alignment

181 reached a size of 10-12 sequences, we were able to retrieve most of the sequences that made it into the

182 final alignment through further iterations of Infernal searches and manual addition to the alignment. Hits

183 for unclassified viruses were also retrieved from large-scale transcriptomics data of invertebrate and

184 vertebrate-associated RNA viruses, using the deposited sequences ${ }^{40,41}$.

185 A statistical validation of the final proposed alignment of 47 sequences was performed using the latest

186 version of R-scape available at http://eddylab.org/R-scape/ ${ }^{29}$ (last accessed on August 17, 2018). The

187 corresponding conserved structure and sequence patterns were rendered using R2R v. $1.0 .5^{42}$.

189 Design of RNAs for in vitro assays. DNA templates for in vitro transcription were gBlocks ordered from

190 IDT, cloned into pUC19 and verified by sequencing. RNA constructs for Xrn1 degradation assays

191 contained the xrRNA sequence plus $\sim 30$ nucleotides of the endogenous upstream sequence ('leader

192 sequence') to allow loading of the exoribonucleases. Below are the sequences used in in vitro Xrn1

193 degradation assays with the T7 promoter underlined, the leader sequence in italic and the first protected

194 nucleotides (experimentally validated as described below) in bold. Lower case letters indicate extra

195 nucleotides inserted to allow better transcription.

196 OPMV xrRNA:

197 TAATACGACTCACTATAGGAATTGCCTCCACCAGTAACTAAACCCAACCACAGCCAAGCATTAA

198 GTTGCAAGCGTTGGAGTGGCAGGCTTAACGTCCGACAGTACGACAACTGCGG 


\section{HuPLV1 xrRNA:}

TAATACGACTCACTATAgGCCACAAAACGAATAAAGGAAGAACGCACGAGAGTCAGCCAAACA

RNA preparation. DNA templates for in vitro transcription were amplified by PCR using custom DNA

213 primers (IDT) and Phusion Hot Start polymerase (New England BioLabs). $2.5 \mathrm{~mL}$ transcription reactions

214 were assembled using $1000 \mu \mathrm{L}$ PCR reactions as template ( $\sim 0.2 \mu \mathrm{M}$ template DNA), $6 \mathrm{mM}$ each NTP, 60

$215 \mathrm{mM} \mathrm{MgCl2}, 30 \mathrm{mM}$ Tris $\mathrm{pH}$ 8.0, $10 \mathrm{mM}$ DTT, 0.1\% spermidine, 0.1\% Triton X-100, T7 RNA

216 polymerase and $2 \mu \mathrm{L}$ RNasin RNase inhibitor (Promega) and incubated overnight at $37^{\circ} \mathrm{C}$. After

217 inorganic pyrophosphates were precipitated by centrifugation, the reactions were ethanol precipitated and

218 purified on a $7 \mathrm{M}$ urea $8 \%$ denaturing polyacrylamide gel. RNAs of the correct size were gel-excised,

219 eluted overnight at $4{ }^{\circ} \mathrm{C}$ into $\sim 40 \mathrm{~mL}$ of diethylpyrocarbonate (DEPC)-treated milli-Q filtered water

220 (Millipore) and concentrated using Amicon Ultra spin concentrators (Millipore). Mutations were

221 introduced using mutagenized custom DNA reverse primers.

222 Primers used in this study (mutated residues in bold):

223 OPMV_WT_rev

224 5'-CCGCAGTTGTCGTACTGTCGG-3'

225 OPMV_PKmut1_rev

226 5'-CCGCAGTTGTCGTACTGTCGGACGAATTGCCTGCCACTCCAACGC-3' 
OPMV_PKmut2_rev

228 5'-CCGCAGTTGTCGTACTGTCGGACGTTAAGCCTGCCACTCCAACGCTTGCAACAATTTGCTT 229 GGCTGTGGTTGG-3'

230 OPMV_PKcomp_rev

231 5'-CCGCAGTTGTCGTACTGTCGGACGAATTGCCTGCCACTCCAACGCTTGCAACAATTTGCTT 232 GGCT GTGGTTGG-3'

233 MCMV_WT_rev

234 5'-TGGC̄AGGACTCTGCCAGAAGG-3'

235 MCMV_PKmut1_rev

236 5'-TGGC̈AGGACTCTGCCAGCTCCACTGGTTGTTCCGGTCTTGC-3'

237 MCMV PKmut2 rev

238 5'-TGGCAGGACTCTGCCAGAAGGACTGGTTGTTCCGGTCTTGCAAGGAGATGCCGGCTCACC 239 TTGTGCTC-3'

240 MCMV_PKcomp_rev

241 5'-TGGCAGGACTCTGCCAGCTCCACTGGTTGTTCCGGTCTTGCAAGGAGATGCCGGCTCACC 242 TTGTGCTC-3'

243 PLRV_WT_rev

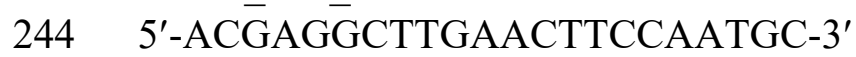

245 PLRV_PKmut1_rev

246 5'-TGCTGGCTTGAACTTCCAATGCTTGC-3'

247 PLRV_PKmut2_rev

248 5'-ACGAGGCTTGAACTTCCAATGCTTGCAACAGCAGTATGCTTGGCTAGTTTTAGTG-3'

249 PLRV_PKcomp_rev

250 5'-TGCTGGCTTGAACTTCCAATGCTTGCAACAGCAGTATGCTTGGCTAGTTTTAGTG-3'

251 MYDV-RMV_WT_rev

252 5'-TATCGAGACTAGGTCATCCAGTGC-3'

253 huPLV_WT_rev

254 5'-ACAAGACTAGAATGAGTCTCC-3'

255 huPLV_PKmut1_rev

256 5'- TGTTGACTAGGATGAGTCTCCAACACTTGC-3'

257 huPLV_PKmut2_rev

258 5' - ACĀAGACTAGAATGAGTCTCCAACACTTGCAACAACAGTTTGTTTGGCTGACTCTCG-3'

259 huPLV_PKcomp_rev

260 5' - TGT'TGACTÄGAATGAGTCTCCAACACTTGCAACAACAGTTTGTTTGGCTGACTCTCG-3'

261 In vitro Xrn1 resistance assays. $4 \mu \mathrm{g}$ RNA was resuspended in $40 \mu \mathrm{L} 100 \mathrm{mM} \mathrm{NaCl}, 10 \mathrm{mM} \mathrm{MgCl}, 50$

$262 \mathrm{mM}$ Tris $\mathrm{pH} 7.5,1 \mathrm{mM}$ DTT and re-folded at $90^{\circ} \mathrm{C}$ for 3 minutes then $20^{\circ} \mathrm{C}$ for 5 minutes. $3 \mu \mathrm{L}$

263 recombinant $\mathrm{RppH}(0.5 \mu \mathrm{g} / \mu \mathrm{L}$ stock) was added and the samples were split into two $20 \mu \mathrm{L}$ reactions (-/+ 
264 exoribonuclease). $1 \mu \mathrm{L}$ of the recombinant $\operatorname{Xrn} 1(0.8 \mu \mathrm{g} / \mu \mathrm{L}$ stock $)$ was added where indicated. All 265 reactions were incubated for $2 \mathrm{hrs}$ at $30^{\circ} \mathrm{C}$ using a thermocycler. The degradation reactions were resolved 266 on a $7 \mathrm{M}$ urea $8 \%$ denaturing polyacrylamide gel and stained with ethidium bromide.

268 Mapping of the exoribonuclease halt site. To determine the Xrn1 stop site at single-nucleotide

resolution, $30 \mu \mathrm{g}$ in vitro-transcribed RNA was degraded using recombinant RppH and Xrn1 as described above (the reaction volume was scaled up to $300 \mu \mathrm{L}$, and $20 \mu \mathrm{L}$ of each enzyme was used). The degradation reaction was resolved on a $7 \mathrm{M}$ urea $8 \%$ polyacrylamide gel, then the Xrn1-resistant degradation product was cut from the gel and eluted overnight at $4{ }^{\circ} \mathrm{C}$ into $\sim 20 \mathrm{~mL}$ of diethylpyrocarbonate (DEPC)-treated milli-Q filtered water (Millipore) and concentrated using Amicon Ultra spin concentrators (Millipore). Once recovered, the RNA was reverse-transcribed using Superscript III reverse transcriptase (Thermo) and a 6-FAM (6-fluorescein amidite)-labeled sequence-specific reverse primer (IDT) with an (A)20 -stretch at the $5^{\prime}$ end to allow cDNA purification with oligo(dT) beads. $10 \mu \mathrm{L}$ buffer, $0.25 \mu \mathrm{L}$ 0.1 M DTT, $0.4 \mu \mathrm{L} 10 \mathrm{mM}$ dNTP mix, $0.1 \mu \mathrm{L}$ Superscript III reverse transcriptase (200 $\mathrm{U} / \mu \mathrm{L})$ and were incubated for 1 hour at $50^{\circ} \mathrm{C}$. To hydrolyze the RNA template after reverse transcription, $5 \mu \mathrm{L}$ of $0.4 \mathrm{M} 4 \mathrm{NaOH}$ was added and the reaction mix incubated at $90^{\circ} \mathrm{C}$ for $3 \mathrm{~min}$, followed by cooling on ice for $3 \mathrm{~min}$. The reaction was neutralized by adding $5 \mu \mathrm{L}$ of acid quench mix $(1.4 \mathrm{M} \mathrm{NaCl}, 0.57 \mathrm{M}$ $\mathrm{HCl}, 1.3 \mathrm{M}$ sodium acetate $\mathrm{pH}$ 5.2), then $1.5 \mu \mathrm{L}$ oligo(dT) beads (Poly(A)Purist MAG Kit (Thermo)) were added and the cDNA purified on a magnetic stand according to the manufacturer's instructions. The cDNA was eluted in $11 \mu \mathrm{L}$ ROX-HiDi and analyzed on a 3500 Genetic Analyzer (Applied Biosystems) 285 for capillary electrophoresis. A Sanger sequencing (ddNTP) ladder of the undigested RNA was analyzed alongside each degradation product as reference for band annotation. 


\section{CONTRIBUTIONS}

288 Q.V., A.-L.S., J.S.K. designed and analyzed research; Q.V. performed the computational search; A.-L.S. 289 performed the biochemical experiments; Q.V., A.-L.S. \& J.S.K wrote the paper.

\section{ACKNOWLEDGEMENTS}

291 We gratefully acknowledge David Farrell for IT support; the Kieft lab members for useful discussions;

292 and W. Allen Miller and David Costantino for careful reading of the manuscript.

\section{FINANCIAL SUPPORT}

294 This work was supported by NIH grants R35GM118070 and R01AI133348 (J.S.K.) and DFG STE 295 2509/2-1 (A.-L.S.). 


\section{REFERENCES}

1 Miller, W. A., Shen, R., Staplin, W. \& Kanodia, P. Noncoding RNAs of Plant Viruses and Viroids: Sponges of Host Translation and RNA Interference Machinery. Mol Plant Microbe Interact 29, 156-164, doi:10.1094/MPMI-10-15-0226-FI (2016).

2 Sztuba-Solińska, J., Stollar, V. \& Bujarski, J. J. Subgenomic messenger RNAs: mastering regulation of (+)-strand RNA virus life cycle. Virology 412, 245-255, doi:10.1016/j.virol.2011.02.007 (2011).

3 Miller, W. A. \& Koev, G. Synthesis of subgenomic RNAs by positive-strand RNA viruses. Virology 273, 1-8, doi:10.1006/viro.2000.0421 (2000).

4 Jiwan, S. D. \& White, K. A. Subgenomic mRNA transcription in Tombusviridae. RNA Biol 8, 287-294 (2011).

5 Koev, G. \& Miller, W. A. A positive-strand RNA virus with three very different subgenomic RNA promoters. J Virol 74, 5988-5996 (2000).

6 Shen, R. \& Miller, W. A. Subgenomic RNA as a riboregulator: negative regulation of RNA replication by Barley yellow dwarf virus subgenomic RNA 2. Virology 327, 196-205, doi:10.1016/j.virol.2004.06.025 (2004).

7 Flobinus, A. et al. Beet Necrotic Yellow Vein Virus Noncoding RNA Production Depends on a 5'->3' Xrn Exoribonuclease Activity. Viruses 10, doi:10.3390/v10030137 (2018).

8 Pijlman, G. P. et al. A highly structured, nuclease-resistant, noncoding RNA produced by flaviviruses is required for pathogenicity. Cell Host Microbe 4, 579-591, doi:10.1016/j.chom.2008.10.007 (2008).

9 Roby, J. A., Pijlman, G. P., Wilusz, J. \& Khromykh, A. A. Noncoding subgenomic flavivirus RNA: multiple functions in West Nile virus pathogenesis and modulation of host responses. Viruses 6, 404-427, doi:10.3390/v6020404 (2014).

10 Iwakawa, H. O. et al. A viral noncoding RNA generated by cis-element-mediated protection against 5'->3' RNA decay represses both cap-independent and cap-dependent translation. J Virol 82, 10162-10174, doi:10.1128/JVI.01027-08 (2008).

11 Chapman, E. G., Moon, S. L., Wilusz, J. \& Kieft, J. S. RNA structures that resist degradation by Xrn1 produce a pathogenic Dengue virus RNA. Elife 3, e01892, doi:10.7554/eLife.01892 (2014).

12 Moon, S. L. et al. A noncoding RNA produced by arthropod-borne flaviviruses inhibits the cellular exoribonuclease XRN1 and alters host mRNA stability. RNA 18, 2029-2040, doi:10.1261/rna.034330.112 (2012).

13 Charley, P. A., Wilusz, C. J. \& Wilusz, J. Identification of phlebovirus and arenavirus RNA sequences that stall and repress the exoribonuclease XRN1. J Biol Chem 293, 285-295, doi:10.1074/jbc.M117.805796 (2018).

14 Clarke, B. D., Roby, J. A., Slonchak, A. \& Khromykh, A. A. Functional non-coding RNAs derived from the flavivirus 3' untranslated region. Virus Res 206, 53-61, doi:10.1016/j.virusres.2015.01.026 (2015).

15 Schnettler, E. et al. Induction and suppression of tick cell antiviral RNAi responses by tick-borne flaviviruses. Nucleic Acids Res 42, 9436-9446, doi:10.1093/nar/gku657 (2014).

16 Schnettler, E. et al. Noncoding flavivirus RNA displays RNA interference suppressor activity in insect and Mammalian cells. J Virol 86, 13486-13500, doi:10.1128/JVI.01104-12 (2012).

17 Göertz, G. P. et al. Noncoding Subgenomic Flavivirus RNA Is Processed by the Mosquito RNA Interference Machinery and Determines West Nile Virus Transmission by Culex pipiens Mosquitoes. J Virol 90, 10145-10159, doi:10.1128/JVI.00930-16 (2016).

18 Schuessler, A. et al. West Nile virus noncoding subgenomic RNA contributes to viral evasion of the type I interferon-mediated antiviral response. J Virol 86, 5708-5718, doi:10.1128/JVI.0020712 (2012). 
19 Bidet, K., Dadlani, D. \& Garcia-Blanco, M. A. G3BP1, G3BP2 and CAPRIN1 are required for translation of interferon stimulated mRNAs and are targeted by a dengue virus non-coding RNA. PLoS Pathog 10, e1004242, doi:10.1371/journal.ppat.1004242 (2014).

20 Liu, Y., Liu, H., Zou, J., Zhang, B. \& Yuan, Z. Dengue virus subgenomic RNA induces apoptosis through the Bcl-2-mediated PI3k/Akt signaling pathway. Virology 448, 15-25, doi:10.1016/j.virol.2013.09.016 (2014).

21 Manokaran, G. et al. Dengue subgenomic RNA binds TRIM25 to inhibit interferon expression for epidemiological fitness. Science 350, 217-221, doi:10.1126/science.aab3369 (2015).

22 Akiyama, B. M. et al. Zika virus produces noncoding RNAs using a multi-pseudoknot structure that confounds a cellular exonuclease. Science 354, 1148-1152, doi:10.1126/science.aah3963 (2016).

23 Chapman, E. G. et al. The structural basis of pathogenic subgenomic flavivirus RNA (sfRNA) production. Science 344, 307-310, doi:10.1126/science.1250897 (2014).

24 Funk, A. et al. RNA structures required for production of subgenomic flavivirus RNA. $J$ Virol 84, 11407-11417, doi:10.1128/JVI.01159-10 (2010).

25 MacFadden, A. et al. Mechanism and structural diversity of exoribonuclease-resistant RNA structures in flaviviral RNAs. Nat Commun 9, 119, doi:10.1038/s41467-017-02604-y (2018).

26 Steckelberg, A. L. et al. A folded viral noncoding RNA blocks host cell exoribonucleases through a conformationally dynamic RNA structure. Proc Natl Acad Sci U S A 115, 6404-6409, doi:10.1073/pnas.1802429115 (2018).

27 Nawrocki, E. P. \& Eddy, S. R. Computational identification of functional RNA homologs in metagenomic data. RNA Biol 10, 1170-1179, doi:10.4161/rna.25038 (2013).

28 Lim, C. S. \& Brown, C. M. Know Your Enemy: Successful Bioinformatic Approaches to Predict Functional RNA Structures in Viral RNAs. Front Microbiol 8, 2582, doi:10.3389/fmicb.2017.02582 (2017).

29 Rivas, E., Clements, J. \& Eddy, S. R. A statistical test for conserved RNA structure shows lack of evidence for structure in lncRNAs. Nat Methods 14, 45-48, doi:10.1038/nmeth.4066 (2017).

30 Scheets, K. Maize chlorotic mottle machlomovirus expresses its coat protein from a 1.47-kb subgenomic RNA and makes a 0.34-kb subgenomic RNA. Virology 267, 90-101, doi:10.1006/viro.1999.0107 (2000).

31 Smirnova, E. et al. Discovery of a Small Non-AUG-Initiated ORF in Poleroviruses and Luteoviruses That Is Required for Long-Distance Movement. PLoS Pathog 11, e1004868, doi:10.1371/journal.ppat.1004868 (2015).

32 Ryabov, E. V. et al. Genus: Umbravirus. 1191-1195 (International Committee on Taxonomy of Viruses, 2012).

33 Stevens, M., Freeman, B., Liu, H. Y., Herrbach, E. \& Lemaire, O. Beet poleroviruses: close friends or distant relatives? Mol Plant Pathol 6, 1-9, doi:10.1111/j.1364-3703.2004.00258.X (2005).

34 Miller, W. A., Jackson, J. \& Feng, Y. Cis- and trans-regulation of luteovirus gene expression by the 3' end of the viral genome. Virus Res 206, 37-45, doi:10.1016/j.virusres.2015.03.009 (2015).

35 Miller, W. A. \& White, K. A. Long-distance RNA-RNA interactions in plant virus gene expression and replication. Annu Rev Phytopathol 44, 447-467, doi:10.1146/annurev.phyto.44.070505.143353 (2006).

36 Newburn, L. R. \& White, K. A. Cis-acting RNA elements in positive-strand RNA plant virus genomes. Virology 479-480, 434-443, doi:10.1016/j.virol.2015.02.032 (2015).

37 Johnston, J. C. \& Rochon, D. M. Deletion analysis of the promoter for the cucumber necrosis virus 0.9-kb subgenomic RNA. Virology 214, 100-109, doi:10.1006/viro.1995.9950 (1995).

38 Toor, N., Keating, K. S., Taylor, S. D. \& Pyle, A. M. Crystal structure of a self-spliced group II intron. Science 320, 77-82, doi:10.1126/science.1153803 (2008). 
39439 Okonechnikov, K., Golosova, O., Fursov, M. \& team, U. Unipro UGENE: a unified

395 bioinformatics toolkit. Bioinformatics 28, 1166-1167, doi:10.1093/bioinformatics/bts091 (2012).

39640 Shi, M. et al. The evolutionary history of vertebrate RNA viruses. Nature 556, 197-202, 397 doi:10.1038/s41586-018-0012-7 (2018).

39841 Shi, M. et al. Redefining the invertebrate RNA virosphere. Nature, doi:10.1038/nature20167 $399 \quad$ (2016).

40042 Weinberg, Z. \& Breaker, R. R. R2R--software to speed the depiction of aesthetic consensus RNA 401 secondary structures. BMC Bioinformatics 12, 3, doi:10.1186/1471-2105-12-3 (2011).

40243 Leontis, N. B. \& Westhof, E. The annotation of RNA motifs. Comp Funct Genomics 3, 518-524, 403 doi:10.1002/cfg.213 (2002). 
bioRxiv preprint doi: https://doi.org/10.1101/433672; this version posted October 2, 2018. The copyright holder for this preprint (which was not certified by peer review) is the author/funder. All rights reserved. No reuse allowed without permission.

\section{TABLE}

407 Table 1. Selected set of plant viruses possessing an $\mathrm{xrRNA}_{\mathrm{TL}}$. Viruses are grouped by their genomic

408 context (last column). The complete list of sequences used for comparative sequence alignment is shown 409 in Table S1.

\begin{tabular}{|c|c|c|c|c|c|c|}
\hline Name & Abbreviation & Classification & GenBank ID & \begin{tabular}{|c|c} 
Total ssRNA \\
length (nt)
\end{tabular} & $\begin{array}{l}\text { Genomic } \\
\text { location* }^{*}\end{array}$ & Genomic context \\
\hline Red clover necrotic mosaic virus & RCNMV & Tombusviridae; Dianthovirus & NC_003756 & 3890 & $3461-3504$ & 3' UTR \\
\hline Sweet clover necrotic mosaic virus & SCNMV & Tombusviridae; Dianthovirus & NC_003806 & 3876 & $3446-3489$ & 3' UTR \\
\hline Maize chlorotic mottle virus (isolate KS1) & MCMV & Tombusviridae; Machlomovirus & NC_003627 & 4437 & $4101-4143$ & 3' UTR \\
\hline Opium poppy mosaic virus (isolate PHEL5235) & OPMV & Tombusviridae; Umbravirus & NC_027710 & 4230 & $3585-3629$ & 3' UTR \\
\hline Carrot mottle mimic umbravirus & CMoMV & Tombusviridae; Umbravirus & NC_001726 & 4201 & $2664-2706$ & $74 \mathrm{nt}$ to AUG from ORF3 \\
\hline Chickpea chlorotic stunt virus & CpCSV & Luteoviridae; Polerovirus & NC_008249 & 5900 & $3489-3534$ & $\begin{array}{l}11 \mathrm{nt} \text { to AUA from ORF3a; } \\
129 \mathrm{nt} \text { to AUG from ORF3-5 }\end{array}$ \\
\hline Cowpea polerovirus 1 (isolate BE167) & CpPV1 & Luteoviridae; Polerovirus & NC_034246 & 5845 & $3380-3425$ & $\begin{array}{l}11 \mathrm{nt} \text { to } C U \mathrm{G} \text { from ORF3a; } \\
129 \mathrm{nt} \text { to AUG from ORF3-5 }\end{array}$ \\
\hline Cotton leafroll dwarf virus & CoLRDV & Luteoviridae; Polerovirus & NC_014545 & 5866 & $3451-3499$ & $\begin{array}{l}13 \mathrm{nt} \text { to CUG from ORF3a; } \\
131 \mathrm{nt} \text { to AUG from ORF3-5 }\end{array}$ \\
\hline Cereal yellow dwarf virus-RPV & CYDV-RPV & Luteoviridae; Polerovirus & NC_004751 & 5723 & $3566-3622$ & $\begin{array}{l}14 \mathrm{nt} \text { to } A U U \text { from ORF3a; } \\
132 \mathrm{nt} \text { to AUG from ORF3-5 }\end{array}$ \\
\hline Maize yellow dwarf virus-RMV (Formerly BYDV) & MYDV-RMV & Luteoviridae; Polerovirus & NC_021484 & 5612 & $3335-3384$ & $\begin{array}{l}14 \mathrm{nt} \text { to } A C G \text { from ORF3a; } \\
132 \mathrm{nt} \text { to AUG from ORF3-5 }\end{array}$ \\
\hline Potato leafroll virus & PLRV & Luteoviridae; Polerovirus & NC_001747 & 5987 & $3509-3557$ & $\begin{array}{l}18 \mathrm{nt} \text { to AUA from ORF3a; } \\
136 \mathrm{nt} \text { to AUG from ORF3-5 }\end{array}$ \\
\hline Hubei polero-like virus 2 (strain QTM26674) & HuPLV2 & Unclassified & NC_033229 & 6083 & $3706-3753$ & $133 \mathrm{nt}$ to AUG from ORF3-5 \\
\hline Beet western yellows luteovirus (strain bwyv-1, isolate 28a) & BWYV & Luteoviridae; Polerovirus & L39983 & 973 & $341-389$ & $135 \mathrm{nt}$ to AUG from ORF3-5 \\
\hline Hubei polero-like virus 1 (strain WHCC118254) & HuPLV1 & Unclassified & NC_032224 & 4213 & $3357-3410$ & $135 \mathrm{nt}$ to AUG from ORF3-5 \\
\hline Sugarcane yellow leaf virus & ScYLV & Luteoviridae; Polerovirus & NC_000874 & 5899 & $3467-3512$ & $136 \mathrm{nt}$ to $A \cup G$ from ORF3-5 \\
\hline Beet western yellows virus & BWYV & Luteoviridae; Polerovirus & NC_004756 & 5666 & $3346-3393$ & $138 \mathrm{nt}$ to $A \cup G$ from ORF3-5 \\
\hline
\end{tabular}

$411 * x r R N A$ boundaries defined as the $1^{\text {st }}$ nucleotide of the P1 stem and the last nucleotide of the pseudoknot. 


\section{FIGURES}
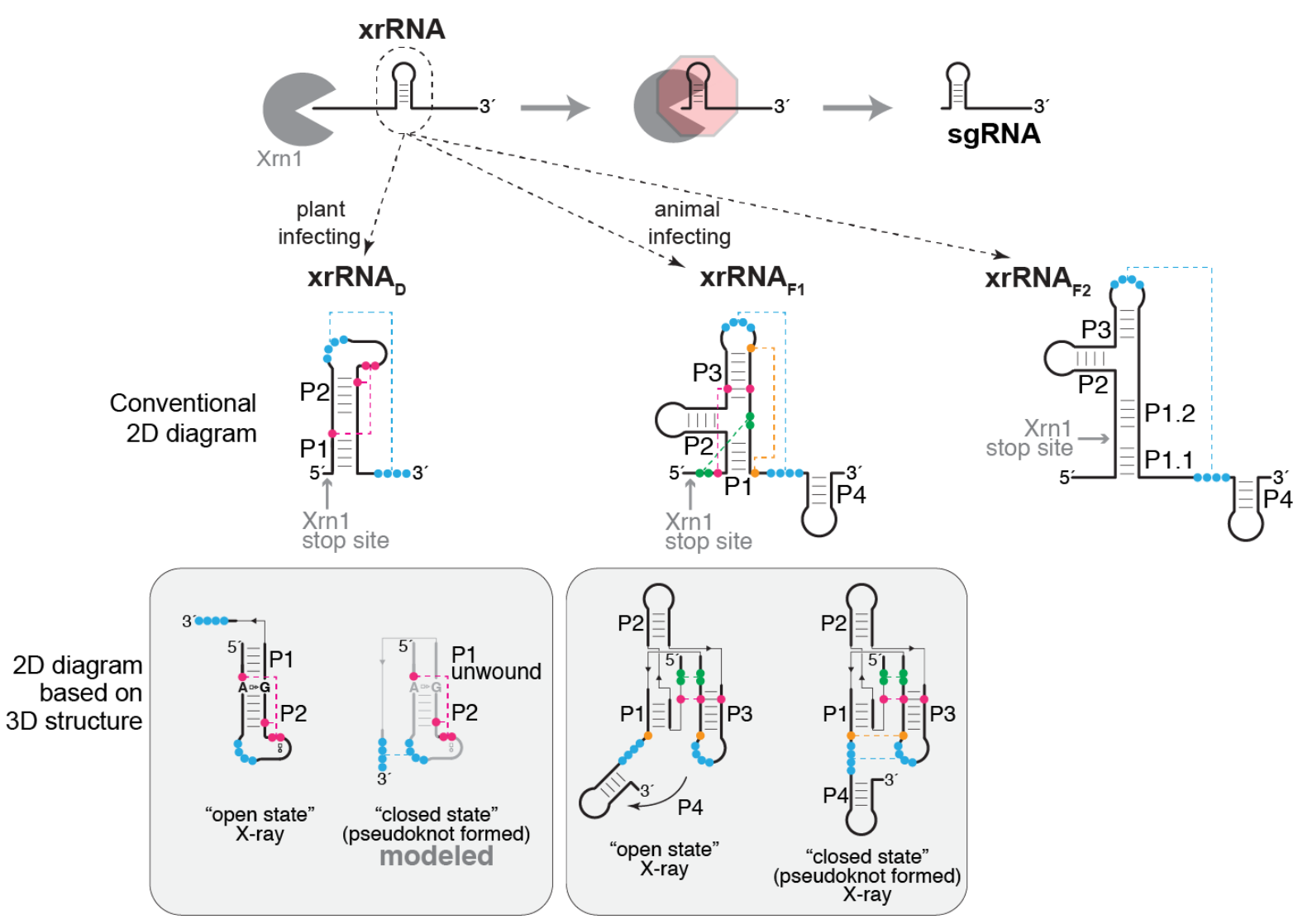

414 Figure 1 | An expanding repertoire of structured RNAs for blocking exoribonuclease degradation.

415 Top: xrRNAs adopt a three-dimensional structure that blocks the progression of 5' to 3' exoribonucleases 416 such as Xrn1 (grey). In the case of flaviviruses and dianthoviruses, xrRNAs are in the 3'UTR and this 417 results in accumulating sgRNAs that comprise the 3'UTR. Middle: Secondary structure diagrams are 418 shown for the two classes of xrRNAs from flaviviruses ( $x r R N A_{F 1}$ and $\left.x r R N A_{F 2}\right)^{15,24,25}$, and from 419 dianthoviruses $\left(x_{R N A}\right)^{26}$. Secondary structure features are labeled, and nucleotides involved in tertiary 420 interactions are shown in colors connected by dashed lines (pseudoknot shown in blue). Experimentally 421 determined Xrn1 stop sites are indicated. Bottom: The grey shaded boxes below each secondary structure 422 contain diagrams reflecting the currently available three-dimensional structures ${ }^{22,23,26}$. The A8-G33 pair 423 is highlighted in the open state of the Sweet clover necrotic mosaic virus (SCNMV) xrRNA. 
a

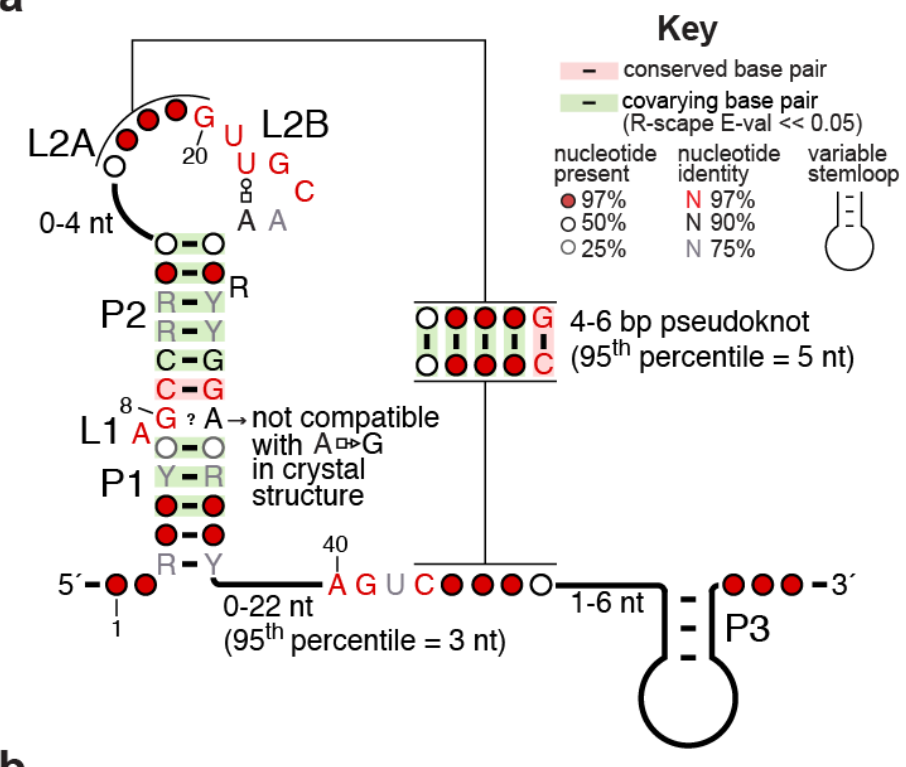

b

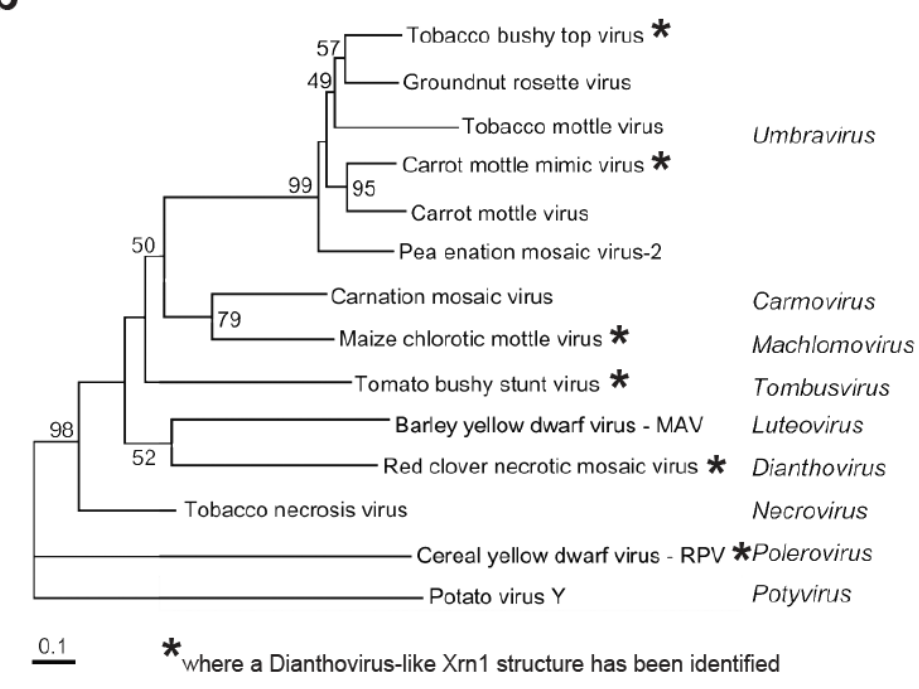

Figure 2 | Widespread occurrence of Xrn1-resistant RNAs among plant viruses. (a) Consensus sequence and secondary structure of $x_{R N A} \mathrm{TL}_{\mathrm{TL}}$ based on a comparative sequence alignment of 47 sequences of viruses belonging to the Tombusviridae and Luteoviridae families (shown in Figure S1). Y = pyrimidine; $\mathrm{R}=$ purine. Non-Watson-Crick base pairs are shown using the Leontis-Westhof nomenclature ${ }^{43}$. The numbering is that of the crystal structure of the SCNMV $\operatorname{xrRNA}_{\mathrm{D}}$ (now referred to as xrRNATL) ${ }^{26}$. (b) Phylogenetic relationship between various plant viruses, based on the RNA-dependent RNA polymerase amino acid sequence ${ }^{32}$. The viruses and corresponding genera in which we identified xrRNA $_{\mathrm{TL}}$ structures are marked by a star. Numbers at the nodes refer to bootstrap values as percentages obtained from 2000 replications, shown only for branches supported by more than $40 \%$. Branch length is proportional to the number of changes. Additional analysis will likely reveal xrRNATL elements in more of these viruses with additional sequence and structural variation. 
A

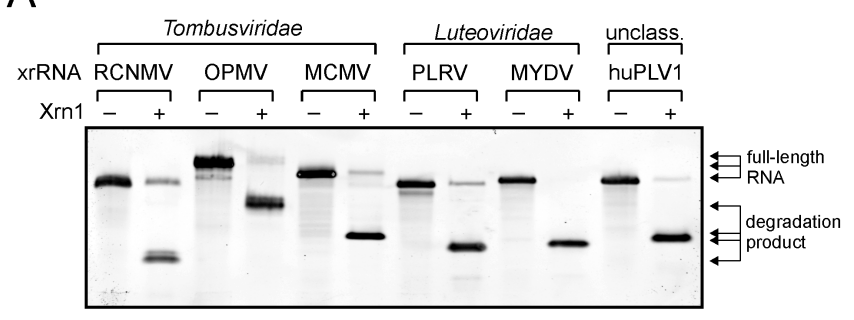

$\mathrm{B}$

\begin{tabular}{|l|l|}
\hline Name & Classification \\
\hline Red clover necrotic mosaic virus (RCNMV) & Tombusviridae, Dianthovirus \\
\hline Opium poppy mosaic virus (OPMV) & Tombusviridae, Umbravirus \\
\hline Maize chlorotic mottle virus (MCMV) & Tombusviridae, Machlomovirus \\
\hline Potato leave roll virus (PLRV) & Luteoviridae, Polerovirus \\
\hline Maize yellow dwarf virus-RMV (MYDV-RMV) & Luteoviridae, Polerovirus \\
\hline Hubei polero-like virus 1 & unclassified \\
\hline
\end{tabular}

C

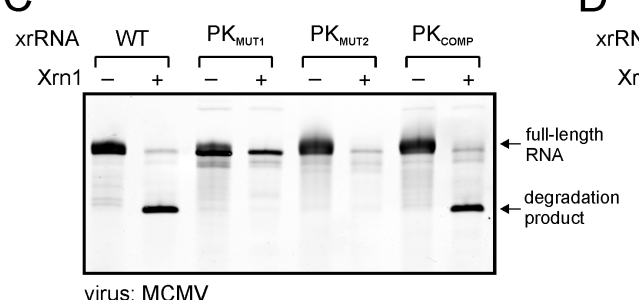

$\mathrm{F}$

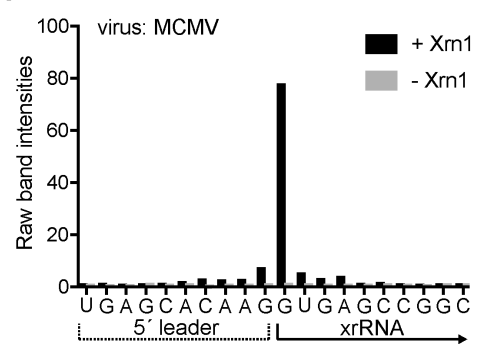

$\mathrm{D}$

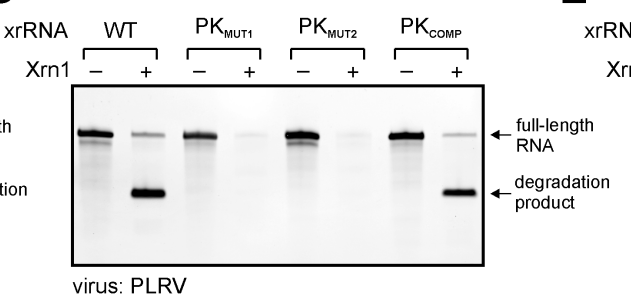

G

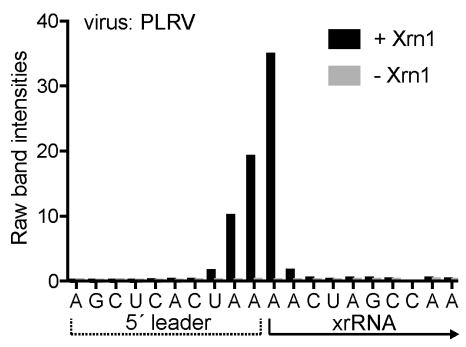

$E$

$\mathrm{H}$
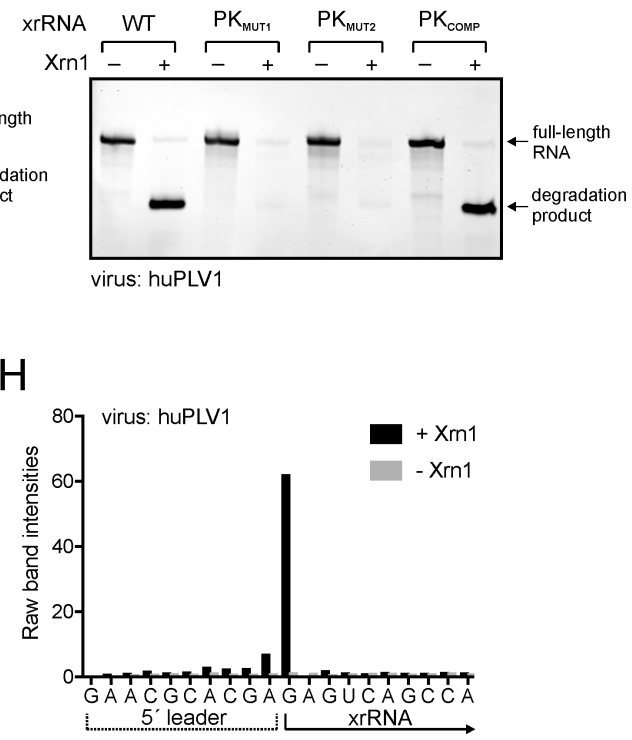

Figure 3 | Biochemical characterization of representative plant virus xrRNA $A_{\text {TL }}$ elements. (A) In vitro Xrn1 resistance assay of putative $x_{R N A}$ Th from various plant RNA viruses (Table 1). The xrRNA from RCNMV was included as a positive control. Arrows indicate the size of full-length RNA and Xrn1resistant degradation product. (B) Classification of viruses used in A (Table 1). (C-E) In vitro Xrn1 resistance assay of WT and PK mutant versions of MCMV (C), PLRV (D) and HuPLV1 (E) xrRNAs. (FH) Reverse transcription (RT) mapping of the Xrn1 halt site. Distribution of RT products of Xrn1resistant fragments of MCMV (F), PLRV (G) and HuPLV1 (H) degradation fragments. Experimentally validated halt sites are indicated on the secondary structure diagram for all tested xrRNATL in Figure S2. 

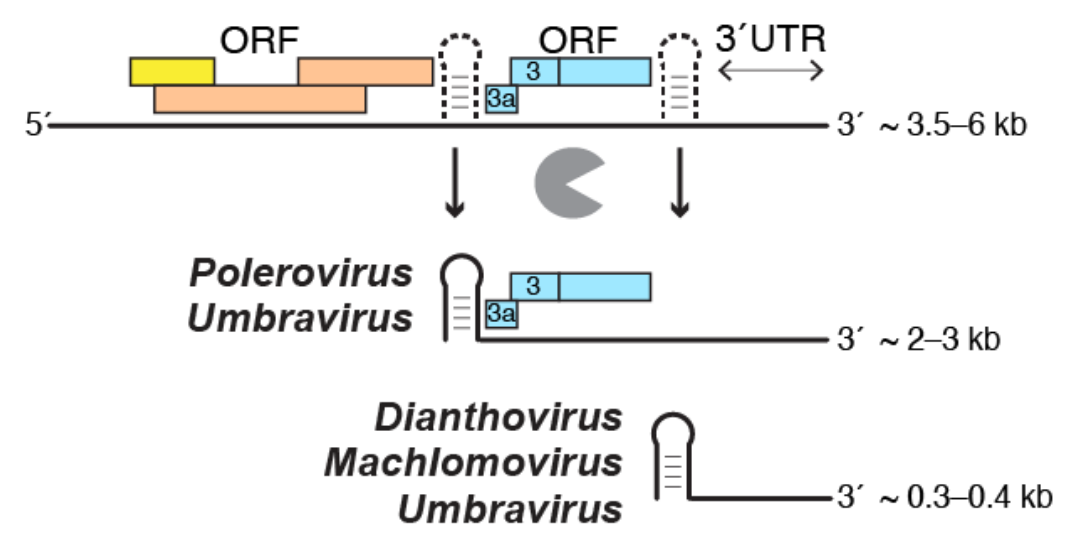

450 Figure $4 \mid$ xrRNA 4 can produce or protect both coding and noncoding sgRNAs. The presence of $451 \mathrm{xrRNA}_{\mathrm{TL}}$ in difference contexts suggests an expanded role for these elements. Shown here, full-length 452 viral genomic RNA (top) could be processed by exonucleases that stop at xrRNAs (depicted as dashed 453 structures) to yield both sgRNAs with protein coding potential (middle) and noncoding sgRNAs (bottom). 454 Also, sgRNAs produced by subgenomic promoters could be "trimmed" or protected by xrRNAs (not 455 shown). Note that only some Umbraviruses (e.g. OPMV) possess two xrRNATL elements. Colored boxes 456 symbolize ORF organization in the plant viruses examined in this study. 